\begin{tabular}{ll}
\hline \hline MINING AND METALLURGY INSTITUTE BOR & ISSN: 2334-8836 \\
& UDK: 622 \\
\hline \hline
\end{tabular}

Dragan Ignjatović, Lidija Djurdjevac Ignjatović, Milenko Ljubojev"

\title{
EFFECT OF DISPLACEMENT THE FLOTATION DAM 2 ON THE ROUTE OF FUTURE COLLECTOR OF THE KRIVELJ RIVER, TESTED USING THE SOFTWARE PHASE2 v8.0**
}

\begin{abstract}
The need to expand the capacity of the flotation Field I is necessary due to increase the capacity of the Flotation Plant Veliki Krivelj. Based on the geotechnical data from previous investigations and as a proposal for displacement the existing Dam 2 to increase the capacity of the flotation Field 1, an analysis the effect of flotation and rock mass on the route of the new Krivelj River collector was carried out using the software package Phase 2.

Keywords: stress condition, flotation dam, Krivelj River's collector, software Phase 2
\end{abstract}

\section{INTRODUCTION}

Due to the increased production volume at the open pit Veliki Krivelj, the amount of flotation tailings increased that has to be disposed. The existing flotation tailing dump becomes small by the capacity to receive the new quantities of material. Due to this reason, it is necessary to find a technicaltechnological solution that is transient and should allow the smooth operation of the Flotation plant Veliki Krivelj for the period of 3 to 4 years, depending on the concept of tailing dump that is adopted.

There is an urgent need to approach the planning and development of the new tailingdump to solve the problem of disposal of tailings long-term, especially as the Flotation Plant Veliki Krivelj processes over
13 million tonnes of ore per year $(10.6+2.5$ Cerovo).

The possibilities were discussed of expanding the capacity of the existing tailing dump in the valley of the Krivelj River, downstream of the Flotation Plant.

From the Flotation Plant Veliki Krivelj to the location of tailing dump, the transport of tailings is done by gravity through a concrete channel, length of 9 $\mathrm{km}$. Transport of tailings from the concrete channel to the sand dams of tailing dump is also gravity and it is performed through the cascades. From cascades, the pulp fed hydrocyclones, placed on the crests of sand dams that are used for separation of sludge and sand.

\footnotetext{
* Mining and Metallurgy Institute Bor, Zeleni bulevar 35, Bor, Serbia

** This work is the result of Project No. 33021 "Research and Monitoring the Changes of Stressstrain State in the Rock Massif "in-situ" Around the Underground Rooms with Development a Model With a Special Review to the Tunnel of the Krivelj River and Jama Bor", funded by the Ministry of Education, Science and Technological Development of the Republic of Serbia
} 


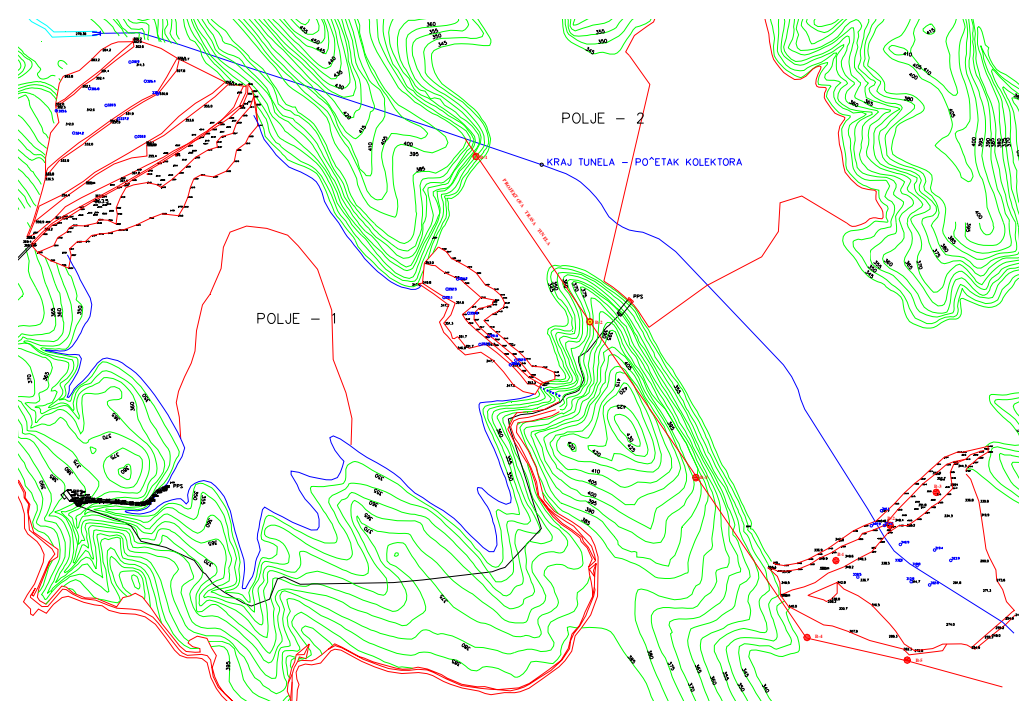

Figure 1 Location map with the new route of collector of the Krivelj River

Currently, 16 batteries of two cyclones are active on dam and they meet the technological needs of Flotation Plant at its full capacity (Krivelj 10.6 million tons and Cerovo 2.5 million tons of run-of-mine ore per year).

Clarified water is returned into technological process as backwater using the floating pump units ( $2 \times 4$ pumps) and relay pumping stations.

Downstream of the village is the active Field 1 which is bounded by dams 1 and 2 .
The final elevation of dams in the Field 1 is 380-384 m.

One of the details of technical-technological solutions that are proposed to increase the capacity of the flotation Field 1 and displacement the flotation Dam 2. To determine what the effect of this displacement of dam is on the future route of the Krivelj River collector, the software Phase 2 was used for the analysis.

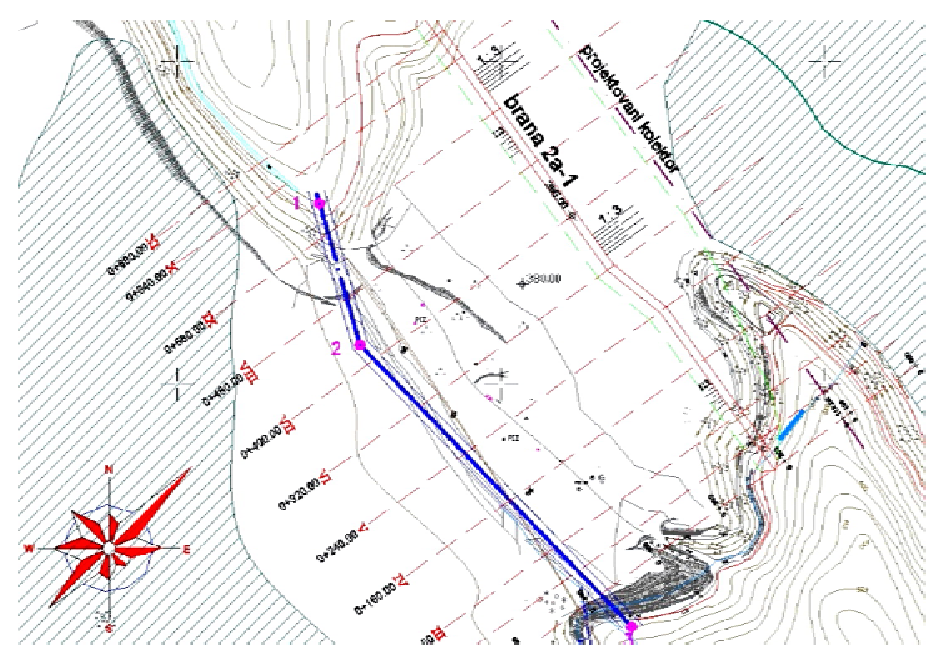

Figure 2 Position of displacement the Dam 2 and profile 7, used in carrying out of all analyses 


\section{STRESS ANALYSES OF \\ CHARACTERISTIC PROFILE VII \\ WITH THE SOFTWARE \\ PHASE 2 v8.0}

PHASE2 v8.0 is 2D program which is based on the stress analysis using the finite element method, for both underground and surface mining of rock or soil. It can be used for a wide range of engineering projects, including the construction of tunnels, slope stability using the finite element method, analysis of groundwater, network modeling, analysis of probabilities and wider. With this program, a complex situation of models in several stage can be quickly created and analyzed.

Values of input parameters per geological units, which are used for calculation on the profile VII-VII are given in Table 1. Parameters of mechanical and deformation properties are taken from the previous geomechanical investigations.

Table 1 Input geomechanical parameters

\begin{tabular}{|c|c|c|c|c|c|c|c|c|}
\hline $\begin{array}{l}\text { Material } \\
\text { Name }\end{array}$ & Color & $\begin{array}{c}\text { Initial } \\
\text { Element } \\
\text { Loading }\end{array}$ & $\begin{array}{c}\text { Unit } \\
\text { weight } \\
\left(\mathrm{kN} / \mathrm{m}^{3}\right)\end{array}$ & $\begin{array}{l}\text { Elastic } \\
\text { Type }\end{array}$ & $\begin{array}{l}\text { Young's } \\
\text { Modulus } \\
\text { (kPa) }\end{array}$ & $\begin{array}{c}\text { Poisson's } \\
\text { Ratio }\end{array}$ & $\begin{array}{c}\text { Failure } \\
\text { Criterion }\end{array}$ & $\begin{array}{c}\text { Material } \\
\text { Type }\end{array}$ \\
\hline $\begin{array}{l}\text { Hydrocyclone } \\
\text { overflow }\end{array}$ & & $\begin{array}{l}\text { Field stress } \\
\text { and body force }\end{array}$ & 18 & Isotropic & 20.000 & 0,47 & $\begin{array}{c}\text { Mohr } \\
\text { Coulomb }\end{array}$ & Plastic \\
\hline $\begin{array}{l}\text { Hydrocyclone } \\
\text { underflow }\end{array}$ & & $\begin{array}{l}\text { Field stress } \\
\text { and body force }\end{array}$ & 23 & Isotropic & 50.000 & 0,40 & $\begin{array}{c}\text { Mohr } \\
\text { Coulomb }\end{array}$ & Plastic \\
\hline $\begin{array}{l}\text { Degraded } \\
\text { andesite }\end{array}$ & & $\begin{array}{l}\text { Field stress } \\
\text { and body force }\end{array}$ & 25 & Isotropic & 550.000 & 0,38 & $\begin{array}{c}\text { Mohr } \\
\text { Coulomb }\end{array}$ & Plastic \\
\hline $\begin{array}{l}\text { Waste rock } \\
\text { from the open } \\
\text { pit }\end{array}$ & & $\begin{array}{l}\text { Field stress } \\
\text { and body force }\end{array}$ & 18,92 & Isotropic & 80.000 & 0,33 & $\begin{array}{c}\text { Mohr } \\
\text { Coulomb }\end{array}$ & Plastic \\
\hline Alluvion & & $\begin{array}{l}\text { Field stress } \\
\text { and body force }\end{array}$ & 20 & Isotropic & 70.000 & 0,40 & $\begin{array}{c}\text { Mohr } \\
\text { Coulomb }\end{array}$ & Plastic \\
\hline
\end{tabular}

continuation of Table 1

\begin{tabular}{|l|c|c|c|c|c|c|c|c|}
\hline \multicolumn{1}{|c|}{$\begin{array}{c}\text { Material } \\
\text { Name }\end{array}$} & Color & $\begin{array}{c}\text { Tensile } \\
\text { Strength } \\
(\mathbf{k P a})\end{array}$ & $\begin{array}{c}\text { Tensile } \\
\text { Strength } \\
\text { residual } \\
(\mathbf{k P a})\end{array}$ & $\begin{array}{c}\text { Dilation } \\
\text { Angle } \\
(\mathbf{d e g})\end{array}$ & $\begin{array}{c}\text { Friction } \\
\text { Angle } \\
\mathbf{p e a k} \\
\mathbf{( d e g}\end{array}$ & $\begin{array}{c}\text { Friction } \\
\text { Angle } \\
\text { residual } \\
(\mathbf{d e g})\end{array}$ & $\begin{array}{c}\text { Cohesion } \\
\text { peak } \\
(\mathbf{k P a})\end{array}$ & $\begin{array}{c}\text { Cohesion } \\
\text { residual } \\
(\mathbf{k P a})\end{array}$ \\
\hline $\begin{array}{l}\text { Hydrocyclone } \\
\text { overflow }\end{array}$ & $\square$ & 0 & 0 & 0 & 25 & 25 & 0 & 0 \\
\hline $\begin{array}{l}\text { Hydrocyclone } \\
\text { underflow }\end{array}$ & $\square$ & 5 & 0 & 0 & 20 & 20 & 5 & 5 \\
\hline $\begin{array}{l}\text { Degraded } \\
\text { andesite }\end{array}$ & $\square$ & 50 & 0 & 0 & 27 & 27 & 50 & 50 \\
\hline $\begin{array}{l}\text { Waste rock } \\
\text { from the open } \\
\text { pit }\end{array}$ & $\square$ & 0 & 0 & 0 & 30 & 30 & 0 & 0 \\
\hline Alluvion & $\square$ & 0 & 0 & 0 & 20 & 20 & 0 & 0 \\
\hline
\end{tabular}

Profile VII was analyzed in two situations:

I. Current condition on the field of flotation Dam 2 and new route of the Krivelj River collector, and
II. Condition after displacement the flotation Dam 2 and new routes of the Krivelj River collector. 


\section{SITUATION}

DAM 2 PROFILE VII

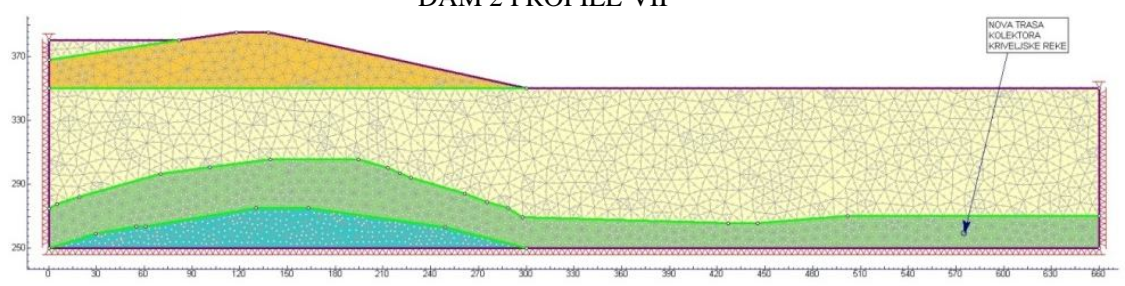

\section{SITUATION}

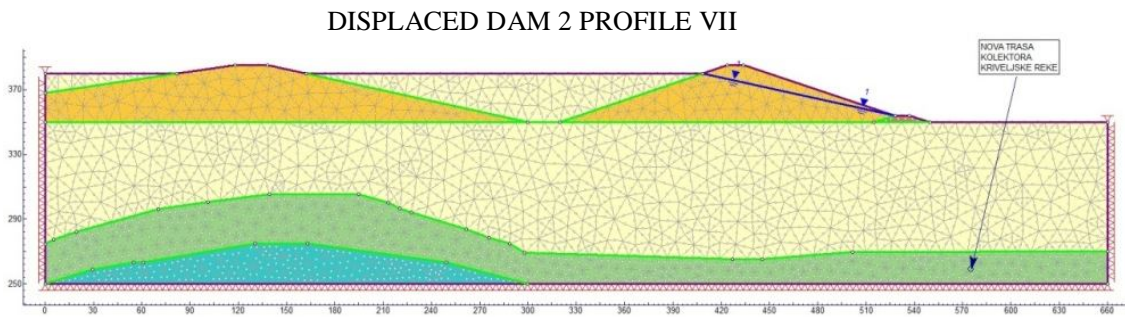

The presented analyses were performed as the ratio of the first and second situation, the relationship between Dam 2 and the new route of the Krivelj River collector. In Figures 3 and 4, an analysis of the profiles on the main normal stress was carried out.

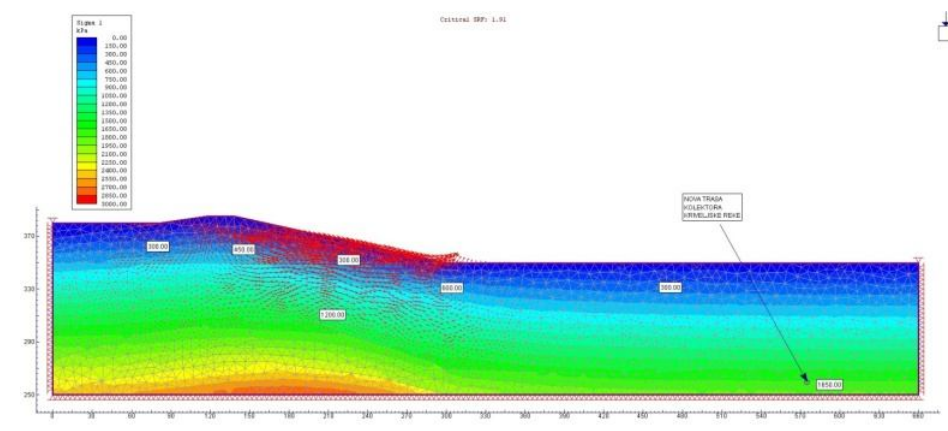

Figure 3 The main normal stress $\sigma_{1}$ in the first situation

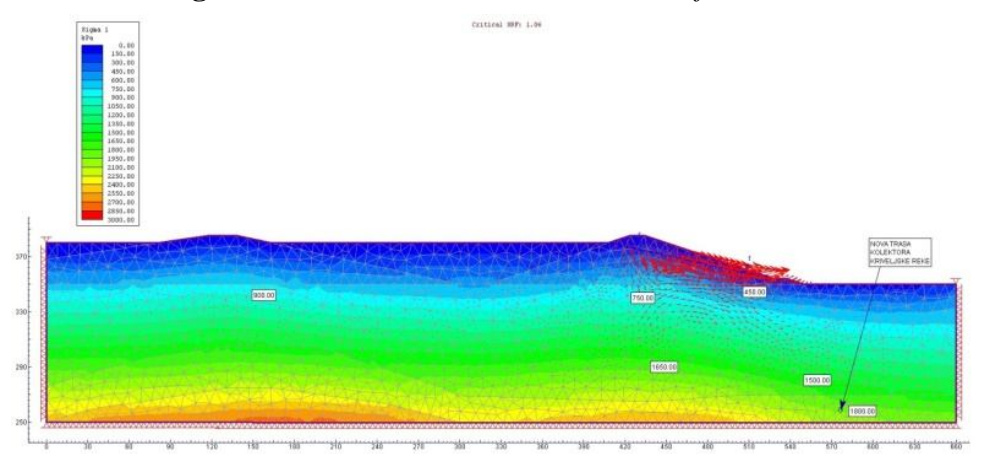

Figure 4 The main normal stress $\sigma_{1}$ in the second situation 
The main normal stress $\sigma_{1}$ in the I situation in the new route of the Krivelj River collector is $1650 \mathrm{kPa}$, and in the II situation is $1800 \mathrm{kPa}$.
In Figures 5 and 6, an analysis of the profile of the main versatile stress was carried out.

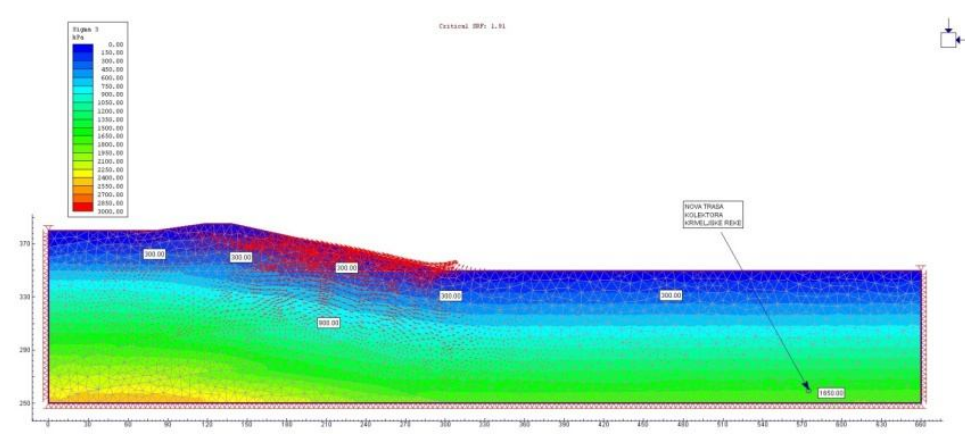

Figure 5 The main versatile stress $\sigma_{3}$ in the I situation

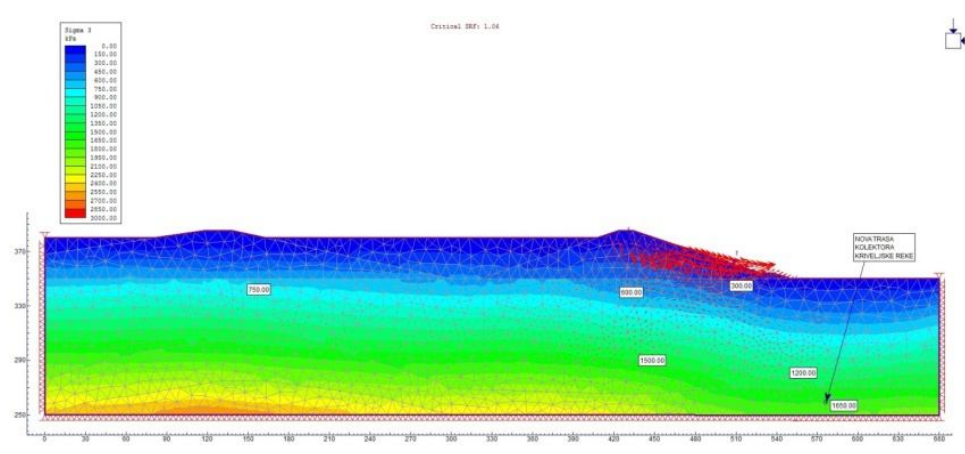

Figure 6 The main versatile stress $\sigma_{3}$ in the II situation

The main versatile stress $\sigma_{3}$ in the I situation in the new route of the Krivelj River collector is $1650 \mathrm{kPa}$, and in the II situation is $1650 \mathrm{kPa}$.
In Figures 7 and 8, an analysis of the profile on differential stress was carried out.

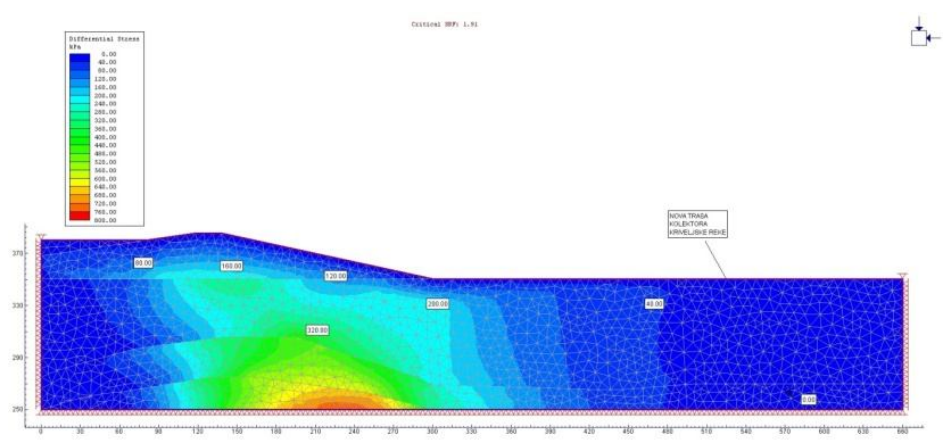

Figure 7 Differential stress $\sigma_{1-3}$ in the I situation 


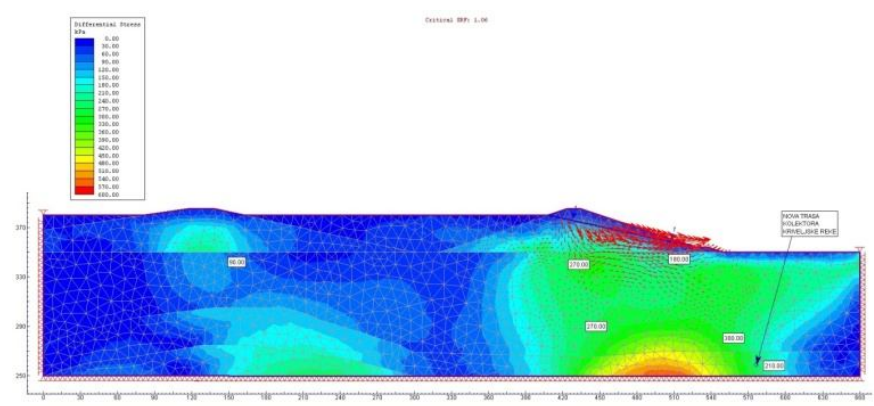

Figure 8 Differential stress $\sigma_{1-3}$ in the II situation

Differential stress $\sigma_{1-3}$ in the I situation in the new route of the Krivelj River collector is $0[\mathrm{kPa}]$, and in the II situation is $210[\mathrm{kPa}]$.
Table 2 gives a review of the results of obtained stresses in the field of passage the future collector of the Krivelj River.

Table 2 Review of the Results

\begin{tabular}{|c|c|c|}
\hline Stress & I situation & II situation \\
\hline Main normal stress $\sigma_{1}$ & $1650 \mathrm{kPa}$ & $1800 \mathrm{kPa}$ \\
\hline Main versatile stress $\sigma_{3}$ & $1650 \mathrm{kPa}$ & $1650 \mathrm{kPa}$ \\
\hline Differential stress $\sigma_{1-3}$ & $0 \mathrm{kPa}$ & $210 \mathrm{kPa}$ \\
\hline
\end{tabular}

\section{CONCLUSIONS}

Based on the obtained results, it can be concluded that displacement of the flotation Dam 2 has no large effect on the stress conditions that occur just on a part of the field where the future collector of the Krivelj River is located.

Despite these results, it is necessary to carry out a continuous monitoring of the field of future route of the Krivelj River collector, as well as displacement route of the flotation Dam 2.

\section{REFERENCES}

[1] GEA Ltd. Enterprise for Design and Engineering Pančevo, Feasibility Study of Expansion the Field I of the Tailing Dump of the Flotation Plant Veliki Krivelj, Pančevo, October 2013 (in Serbian);

[2] Software package PHASE2 v8.0;
[3] M. Ljubojev, R. Popović, D. Rakić, Development of Dynamic Phenomena in the Rock Mass, Mining Engineering, Bor, 1/2011; pp. 109-116

[4] R. Popović, M. Ljubojev, L. Djurdjevac Ignjatović, Deformability Parameters for Formation the Model of Stress-strain State of Rock Mass, Mining and Metallurgy Engineering Bor, 3/2013, pp. 1-6.

[5] S. Ćosić, M. Avdić, A. Sušić, M. Ljubojev, Finite Element Analysis of Deep Underground Salt Caverns, Mining and Metallurgy Engineering Bor, 3/2013, pp. 65-72.

[6] G. Hadži-Niković, S. Ćorić, J. Gomilanović, Application of 3D Slope Stability Analysis in Defining the Excavation Conditions of Coal at the Open Pit, Mining and Metallurgy Engineering Bor, 2/2013, pp. 1-10. 


\begin{tabular}{ll}
\hline \hline INSTITUT ZA RUDARSTVO I METALURGIJU BOR & ISSN: 2334-8836 \\
& UDK: 622 \\
\hline \hline
\end{tabular}

Dragan Ignjatović, Lidija Đurđevac Ignjatović, Milenko Ljubojev **

\section{UTICAJ POMERANJA FLOTACIJSKE BRANE 2 NA TRASU BUDUĆEG KOLEKTORA KRIVELJSKE REKE, TESTIRAN POMOĆU SOFTVERA PHASE2 v8.0**}

Izvod

Potreba proširenja kapaciteta flotacijskog Polja I je neophodna zbog povećanja kapaciteta flotacije Veliki Krivelj. Na osnovu dobijenih geomehaničkih podataka iz ranijih istraživanja kao i predlog pomeranja postojeće brane 2 radi povećanja kapaciteta flotacijskog polja 1, pomoću softverskog paketa Phase2 izvršena je analiza uticaja flotacijske i stenske mase na novu trasu kolektora Kriveljske reke.

Ključne reči: naponsko stanje, flotacijska brana, kriveljski kolektor, softver Phase 2

\section{UVOD}

Zbog povećanja obima proizvodnje na površinskom kopu Veliki Krivelj, povećala se i količina flotacijske jalovine koju treba odložiti. Postojeće flotacijsko jalovište postaje malo po kapacitetu da primi nove količine materijala. Iz tog razloga je potrebno je naći tehničko tehnološko rešenje koje je prelazno i treba da omogući nesmetan rad flotacije Veliki Krivelj za period od 3 do 4 godine, zavisno od koncepta jalovišta koji se usvoji.

Hitno je potrebno pristupiti planiranju i izradi novog jalovišta kako bi se problem odlaganja jalovine dugoročno rešio, pogotovo što Kriveljska flotacija prerađuje preko
13 miliona tona rude godišnje (10,6 Krivelj + 2,5 Cerovo).

Razmatrane su mogućnosti za proširenje kapaciteta postojećeg jalovišta u dolini Kriveljske reke, nizvodno od flotacije.

Od flotacije "Veliki Krivelj" do lokacije jalovišta transport jalovine se obavlja gravitacijski kroz betonski kanal dužine 9 $\mathrm{km}$. Transport jalovine od betonskog kanala do peščanih brana jalovišta je takođe gravitacijski i obavlja se posredstvom kaskada. Iz kaskada pulpa napaja hidrociklone smeštene na krunama peščanih brana u kojima se vrši razdvajanje na mulj i pesak.

\footnotetext{
* Institut za rudarstvo i metalurgiju Bor, Zeleni bulevar 35, Bor, Srbija

** Ovaj rad je proistekao iz. Projekta nauke broj 33021 „Istraživanje i praćenje promena naponsko deformacionog stanja u stenskom masivu "in-situ“ oko podzemnih prostorija sa izradom modela sa posebnim osvrtom na tunel Kriveljske reke i Jame Bor", koga finansira Ministarstvo prosvete, nauke i tehnološkog razvoja Republike Srbije
} 


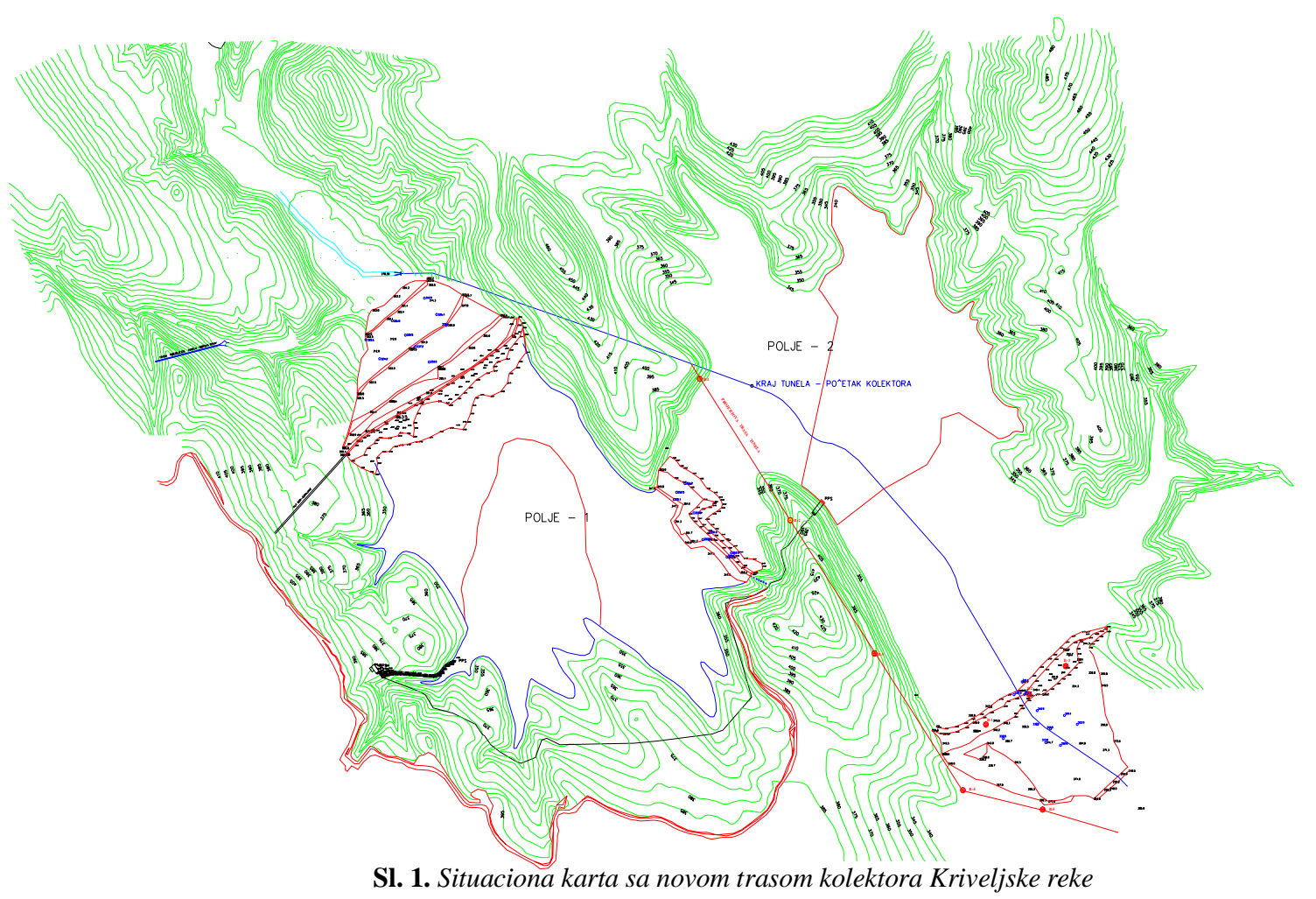

Trenutno na brani je aktivno 16 baterija od po dva ciklona i one zadovoljavaju tehnološke potrebe flotacije pri njenom punom kapacitetu (Krivelj 10,6 miliona tona i Cerovo 2,5 miliona tona rovne rude godišnje).

Izbistrena voda se pomoću plovečih pumpnih agregata ( $2 \times 4$ pumpe) i relejne pumpne stanice vraća u tehnološki proces kao povratna voda.
Nizvodno od sela nalazi se aktivno polje 1 koje je omeđeno branama 1 i 2 . Završna kota brana na polju 1 je 380 - 384 m.

Jedno od detalja tehničko tehnoloških rešenja koja se predlažu za povećanje kapaciteta flotacijskog polja 1 je i pomeranje flotacijske brane 2. Da bi se utvrdilo koliki je uticaj tog pomeranja brane na buduću trasu kolektora Kriveljske reke, za analizu je upotrebljen softver Phase 2.

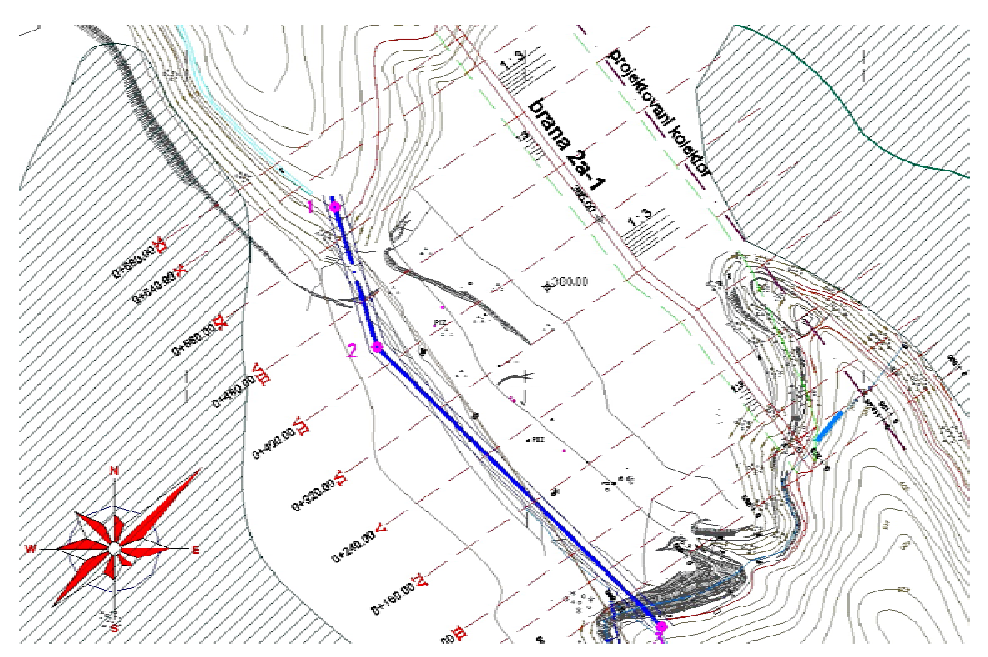

SI. 2. Pozicija pomeranja brane 2 i profil 7 po kome su vršene sve analize 


\section{NAPONSKE ANALIZE}

\section{KARAKTERISTIČNOG PROFILA} VII SA SOFTVEROM PHASE 2 V8.0

PHASE2 v8.0 je 2D program koji svoj rad bazira na analizi napona uz pomoć metode konačnih elemenata, kako za podzemna tako i za površinska otkopavanja stena ili tla. Može se koristiti za široki spektar inženjerskih projekata, uključujući konstrukcije tunela, stabilnost kosina pomoću metode konačnih elemenata, analiza podzemnih voda, mrežno modeliranje, ana- liza verovatnoće i šire. Sa ovim programom se može brzo kreirati i analizirati kompleksno stanje modela u više faza.

Vrednosti ulaznih parametara po geološkim celinama, koji su korišćeni za proračun na profilu VII-VII su dati u tabeli 1 . Parametri mehaničkih i deformacionih osobina su preuzeti iz ranijih geomehaničkih istraživanja.

Tabela 1. Ulazni geomehanički parametri

\begin{tabular}{|c|c|c|c|c|c|c|c|c|}
\hline $\begin{array}{c}\text { Material } \\
\text { Name }\end{array}$ & Color & $\begin{array}{c}\text { Initial } \\
\text { Element } \\
\text { Loading }\end{array}$ & $\begin{array}{c}\text { Unit } \\
\text { weight } \\
\left(\mathrm{kN} / \mathrm{m}^{3}\right)\end{array}$ & $\begin{array}{l}\text { Elastic } \\
\text { Type }\end{array}$ & $\begin{array}{c}\text { Young's } \\
\text { Modulus } \\
\text { (kPa) }\end{array}$ & $\begin{array}{c}\text { Poisson's } \\
\text { Ratio }\end{array}$ & $\begin{array}{l}\text { Failure } \\
\text { Criterion }\end{array}$ & $\begin{array}{c}\text { Material } \\
\text { Type }\end{array}$ \\
\hline $\begin{array}{l}\text { Preliv } \\
\text { hidrociklona }\end{array}$ & & $\begin{array}{l}\text { Field stress } \\
\text { and body force }\end{array}$ & 18 & Isotropic & 20.000 & 0,47 & $\begin{array}{c}\text { Mohr } \\
\text { Coulomb }\end{array}$ & Plastic \\
\hline $\begin{array}{l}\text { Pesak } \\
\text { hidrociklona }\end{array}$ & & $\begin{array}{l}\text { Field stress } \\
\text { and body force }\end{array}$ & 23 & Isotropic & 50.000 & 0,40 & $\begin{array}{c}\text { Mohr } \\
\text { Coulomb }\end{array}$ & Plastic \\
\hline $\begin{array}{l}\text { Degradirani } \\
\text { andezit }\end{array}$ & & $\begin{array}{l}\text { Field stress } \\
\text { and body force }\end{array}$ & 25 & Isotropic & 550.000 & 0,38 & $\begin{array}{c}\text { Mohr } \\
\text { Coulomb }\end{array}$ & Plastic \\
\hline $\begin{array}{l}\text { Jalovina sa } \\
\text { kopa }\end{array}$ & & $\begin{array}{l}\text { Field stress } \\
\text { and body force }\end{array}$ & 18,92 & Isotropic & 80.000 & 0,33 & $\begin{array}{l}\text { Mohr } \\
\text { Coulomb }\end{array}$ & Plastic \\
\hline Aluvion & & $\begin{array}{l}\text { Field stress } \\
\text { and body force }\end{array}$ & 20 & Isotropic & 70.000 & 0,40 & $\begin{array}{c}\text { Mohr } \\
\text { Coulomb }\end{array}$ & Plastic \\
\hline
\end{tabular}

nastavak tabele 1

\begin{tabular}{|l|c|c|c|c|c|c|c|c|}
\hline \multicolumn{1}{|c|}{$\begin{array}{c}\text { Material } \\
\text { Name }\end{array}$} & Color & $\begin{array}{c}\text { Tensile } \\
\text { Strength } \\
(\mathbf{k P a})\end{array}$ & $\begin{array}{c}\text { Tensile } \\
\text { Strength } \\
\text { residual } \\
(\mathbf{k P a})\end{array}$ & $\begin{array}{c}\text { Dilation } \\
\text { Angle } \\
(\mathbf{d e g})\end{array}$ & $\begin{array}{c}\text { Friction } \\
\text { Angle } \\
\mathbf{p e a k} \\
(\mathbf{d e g})\end{array}$ & $\begin{array}{c}\text { Friction } \\
\text { Angle } \\
\text { residual } \\
(\mathbf{d e g})\end{array}$ & $\begin{array}{c}\text { Cohesion } \\
\text { peak } \\
(\mathbf{k P a})\end{array}$ & $\begin{array}{c}\text { Cohesion } \\
\text { residual } \\
(\mathbf{k P a})\end{array}$ \\
\hline $\begin{array}{l}\text { Preliv } \\
\text { hidrociklona }\end{array}$ & $\square$ & 0 & 0 & 0 & 25 & 25 & 0 & 0 \\
\hline $\begin{array}{l}\text { Pesak } \\
\text { hidrociklona }\end{array}$ & $\square$ & 5 & 0 & 0 & 20 & 20 & 5 & 5 \\
\hline $\begin{array}{l}\text { Degradirani } \\
\text { andezit }\end{array}$ & $\square$ & 50 & 0 & 0 & 27 & 27 & 50 & 50 \\
\hline $\begin{array}{l}\text { Jalovina sa } \\
\text { kopa }\end{array}$ & $\square$ & 0 & 0 & 0 & 30 & 30 & 0 & 0 \\
\hline Aluvion & $\square$ & 0 & 0 & 0 & 20 & 20 & 0 & 0 \\
\hline
\end{tabular}

Profil VII analiziran je u dvema sutacijama:

I. Stanje trenutno na terenu flotacijske brane 2 i nove trase kolektora Kriveljske reke i

II. Stanje nakon pomeranja flotacijske brane 2 i nove trase kolektora Kriveljske reke. 


\section{SITUACIJA I}

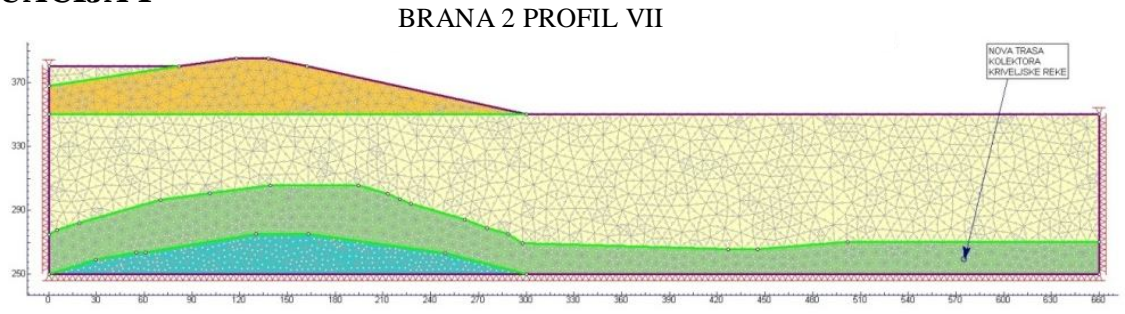

\section{SITUACIJA II}

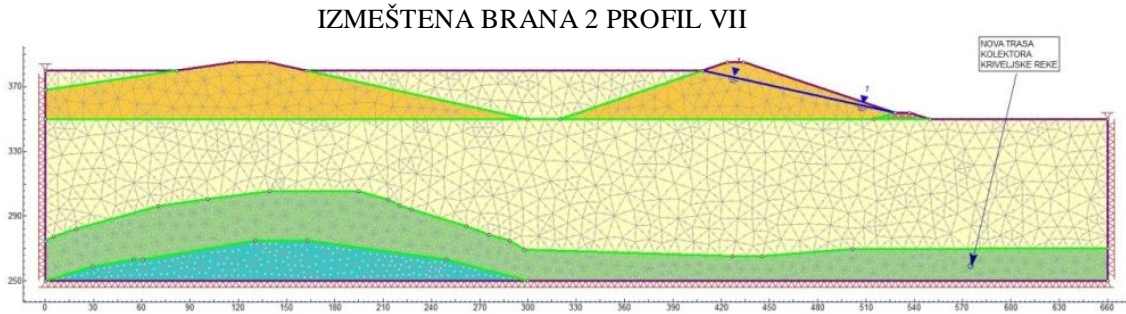

Prikazane analize su rađene kao odnos prve i druge situacije, odnos brane 2 i nove trase kolektora Kriveljske reke.

Na slikama 3 i 4 izvršena je analiza profila na glavni normalni napon.

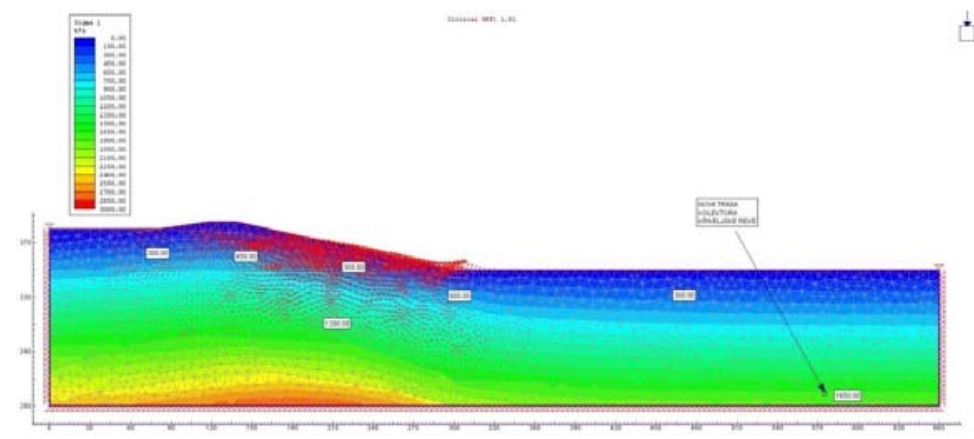

Sl. 3. Glavni normalni napon $\sigma_{1}$ u prvoj situaciji

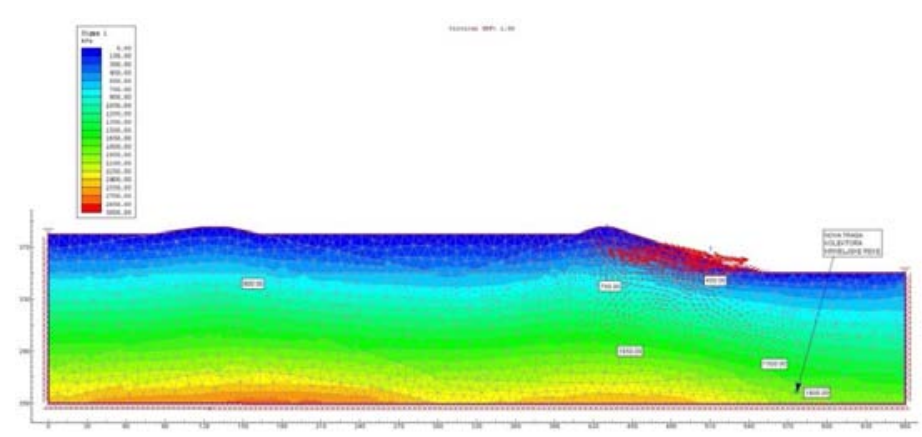

Sl. 4. Glavni normalni napon $\sigma_{1}$ u drugoj situaciji 
Glavni normalni napon $\sigma_{1} \mathrm{u}$ situaciji I kod nove trase kolektora kriveljske reke je $1650 \mathrm{kPa}$, a situacije II $1800 \mathrm{kPa}$.
Na slikama 5 i 6 izvršena je analiza profila na glavni svestrani napon.

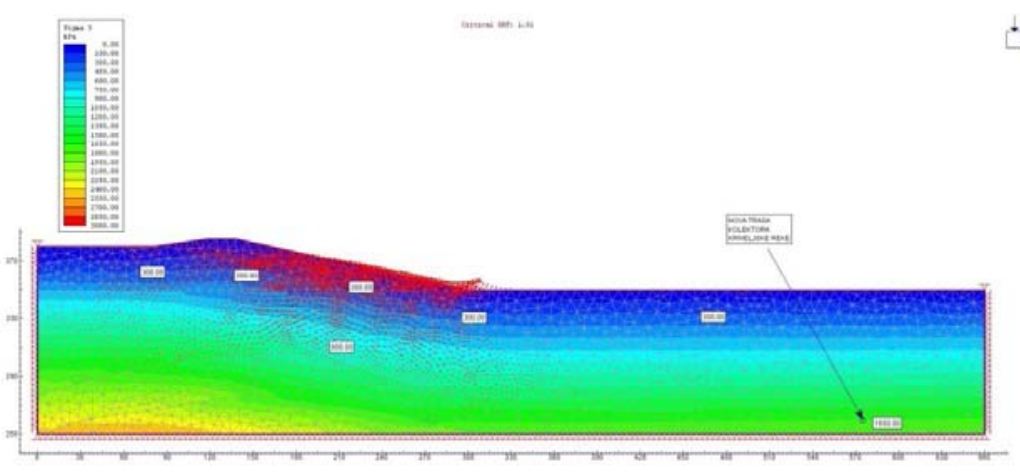

Sl. 5. Glavni svestrani napon $\sigma_{3}$ u prvoj situaciji

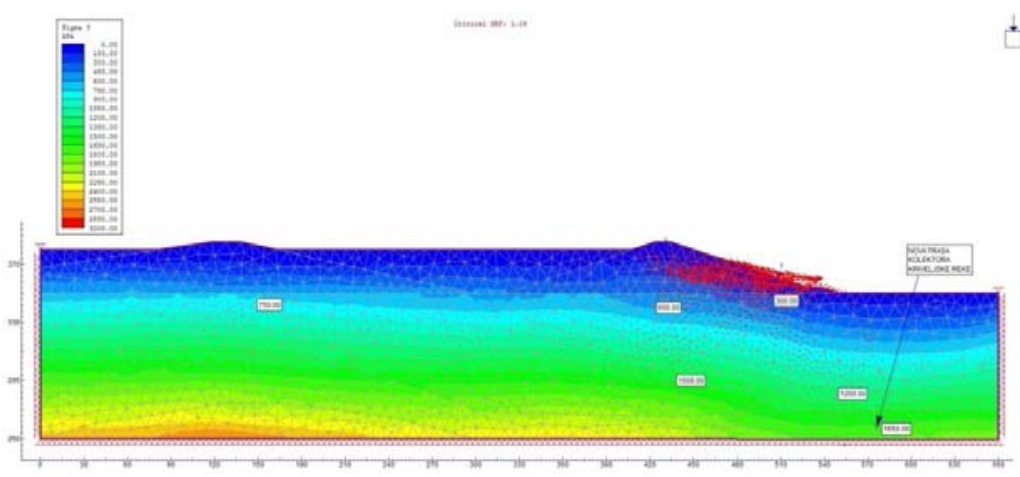

Sl. 6. Glavni svestrani napon $\sigma_{3}$ u drugoj situaciji

Glavni svestrani napon $\sigma_{3} \mathrm{u}$ situaciji I kod nove trase kolektora kriveljske reke je $1650 \mathrm{kPa}$, a situacije II $1650 \mathrm{kPa}$.

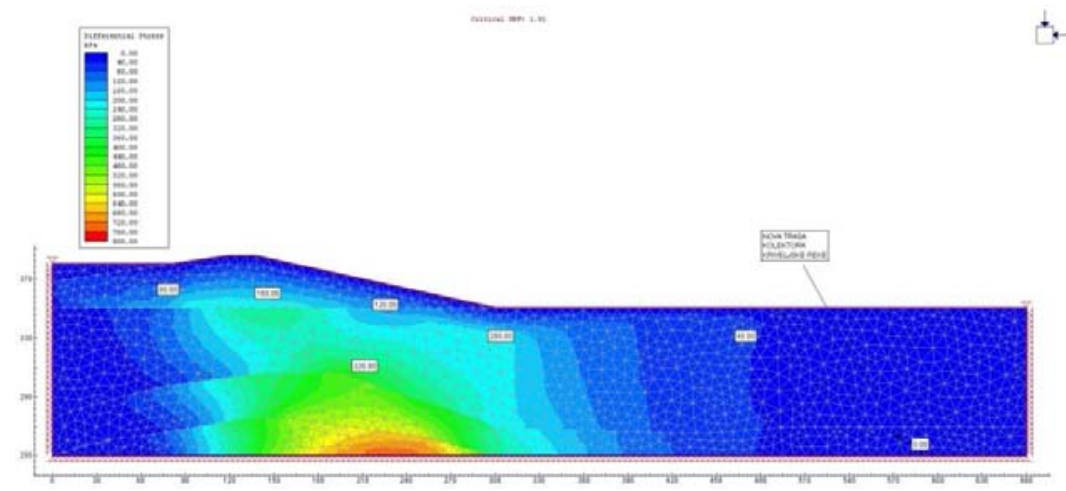

SI. 7. Diferencijalni napon $\sigma_{1-3}$ u prvoj situaciji
Na slikama 7 i 8 izvršena je analiza profila na diferencijalni napon. 


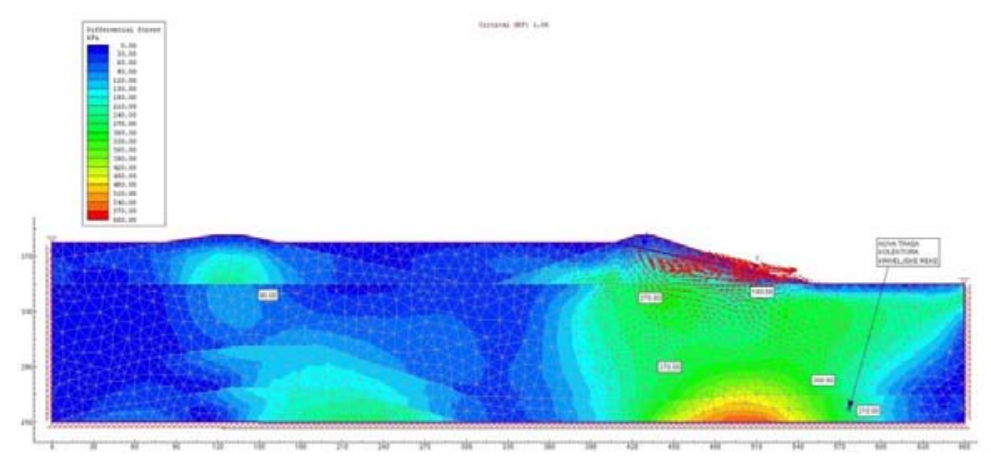

Sl. 8. Diferencijalni napon $\sigma_{l-3} u$ drugoj situaciji

Diferencijalni napon $\sigma_{1-3} \mathrm{u}$ situaciji I kod nove trase kolektora kriveljske reke je $0[\mathrm{kPa}]$ a u situaciji II je $210[\mathrm{kPa}]$.
$\mathrm{U}$ tabeli 2 dat je pregled rezultata dobijenih napona na terenu prolaska budućeg kolektora Kriveljske reke.

Tabela 2. Pregled rezultata

\begin{tabular}{|c|c|c|}
\hline Napon & Situacija I & Situacija II \\
\hline Glavni normalni napon $\sigma_{1}$ & $1650 \mathrm{kPa}$ & $1800 \mathrm{kPa}$ \\
\hline Glavni svestrani napon $\sigma_{3}$ & $1650 \mathrm{kPa}$ & $1650 \mathrm{kPa}$ \\
\hline Diferencijalni napon $\sigma_{1-3}$ & $0 \mathrm{kPa}$ & $210 \mathrm{kPa}$ \\
\hline
\end{tabular}

\section{ZAKLJUČNA RAZMATRANJA}

Na osnovu dobijenih rezultata može se zaključiti da pomeranje flotacijske brane 2 nema velikog uticaja na naponska stanja koja se dešavaju baš na delu terena gde je lociran budući kolektor Kriveljske reke.

I pored ovih rezultata potrebno je vršiti konstantni monitoring terena buduće trase kolektora Kriveljske reke, kao i pomeranje flotacijske brane 2 .

\section{LITERATURA}

[1] GEA d.o.o. Preduzeće za projektovanje i inženjering Pančevo, Studija izvodljivosti proširenja polja I jalovišta pogona Flotacija Veliki Krivelj, Pančevo, oktobar 2013.

[2] Softverski paket PHASE2 v8.0

[3] M. Ljubojev, R. Popović, D. Rakić, Razvoj dinamičkih pojava u stenskoj masi, Rudarski radovi, Bor, 1/2011, str. 101-108

[4] R. Popović, M. Ljubojev, L. Đurđevac Ignjatović, Parametri deformabilnosti za formiranje modela naponsko-deformacijskog stanja stenskog masiva, Mining and Metallurgy Engineering Bor, 3/2013, str. 7-12.

[5] S. Ćosić, M. Avdić, A. Sušić, M. Ljubojev, Naponsko-deformaciona analiza sonih komora metodom konačnih elemenata, Mining and Metallurgy Engineering Bor, 3/2013, str. 73-80.

[6] G. Hadži-Niković, S. Ćorić, J. Gomilanović, Primena 3D analize stabilnosti kosina pri definisanju uslova iskopa uglja u površinskim kopovima, Mining and Metallurgy Engineering Bor, 2/2013, str. 11-20. 